\title{
Research on Model for Evaluating Risks of Venture Capital Projects
}

\author{
Yunfei Li ${ }^{1,2}$ Zongfang Zhou ${ }^{1}$ \\ ${ }^{1}$ School of Management and Economics (University of Electronic Science and Technology of China) \\ Chengdu 610054, China, liyunfei80@126.com ,zhouzf@uestc.edu.cn \\ ${ }^{2}$ School of Mathematics and Information(China West Normal University) \\ Nanchong 637002, China,
}

\begin{abstract}
An index system for evaluating the risks of venture capital projects is established on the basis of analyzing the factors affecting the risks of venture capital projects. A model for evaluating the risk degree of single venture capital project is presented by applying the uncertain type analysis of hierarchy process and the EWAA operator based on the multiple attribute decision making; another model for evaluating the ranks of the risks of multiple venture capital projects is established based on the interval type ideal point for the uncertain multiple attribute decision making. Two examples about practical application are given to show the reasonableness and effectiveness of the models.
\end{abstract}

Keywords: venture capital, risk evaluation, interval number, multiple attribute decision making

\section{基于风险投资项目的风险评价模型研究 李云飞 ${ }^{1,2}$ 周宗放 ${ }^{1}$ \\ 1. 电子科技大学经济与管理学院, 成都 610054 \\ 2. 西华师范大学数学与信息学院, 南充 637002}

摘要：在对风险投资项目的风险因素进行分析的基础上，首先建立了风险投资项目风险评价指标体系， 然后针对单个风险投资项目，综合运用不确定层次分析法（UTAHP）和基于 EWAA 算子的多属性决策方法, 构建了一类评价模型用于其风险等级的确定; 针对多个风险投资项目，构建了一类基于区间型理想点的不确定 多属性决策模型，并应用于实现多个风险投资项目的风险排序。最后给出算例说明了这两个模型的具体应用。

关键词：风险投资，风险评价，区间数，多属性决策方法

\section{1. 引言}

风险投资，又称创业投资，是指向主要属于科 技型的高成长性创业企业（风险项目）提供股权资 本, 并以一定的方式参与所投资创业企业或项目的 管理, 为其提供经营管理和咨询服务, 以期在被投 资企业（风险项目）发展成熟后，通过股权转让获 取中长期资本增值收益的投资行为 ${ }^{[1]}$ 。风险投资的 性质决定了其主要投资对象是具有高度不确定性的 中小型高新技术企业或项目，具有高收益、高风险 的特点 ${ }^{[2]}$ 。风险投资运作的核心是风险投资机构, 风险投资机构是从事风险投资具体运作的组织, 是 连接风险投资者和风险企业家的金融中介。一般而 言，风险投资者（机构投资者和个人投资者）以集 体投资的方式成立风险投资机构（基金），委托风 险投资机构的风险投资家负责风险投资基金的日常 运作与管理。风险投资家在投入资金之前，必须利 用他们长期积累的经验、知识和信息网络对待选的
风险投资项目的风险构成和大小进行分析评价。风 险项目的风险评价结果不仅是风险投资家选择风险 项目的重要依据, 而且直接影响到风险项目运作过 程中的控制权、现金流权的分配，进而影响到风险 投资契约的制定。因此，风险投资项目的风险评价 工作是风险投资家控制与管理风险的前提，直接关 系到风险投资的成败 ${ }^{[3]}$ ，研究风险投资项目的风险 评价问题具有重要的现实意义。

有关风险投资项目的风险评价问题，许多学者 进行了研究, 并取得了一些有价值的研究成果 ${ }^{[2-10]}$ 。 这些成果的研究思路为: 通过对投资项目风险因素 分析的基础上, 构建风险评价指标体系, 然后建立 评价模型进行风险评价。但是其中有些研究所采用 的方法存在不足之处：（1）在确定评价指标权重的 过程中, 有些学者利用传统的层次分析法 (AHP) 对评价指标间的相对重要性程度做判断。然而, 风 险投资项目大多涉及高新技术，其技术、市场、产 品等都不成熟, 具有很大的不确定性, 风险投资家 
由于自身条件的限制或者所掌握的信息不足而对项 目风险因素认识不清, 无法用一个确定的数值表示 风险评估指标两两比较的相对重要性程度。因此, 虽然层次分析法作为一种对非定量事件做定量分析 的实用决策方法, 能有效解决复杂的决策问题, 但 是用一个确定的数表示风险投资项目风险评估指标 间的相对重要性程度, 有失妥当。（2）有些学者采 用模糊综合评估法作为风险评估方法, 但是作为状 态集函数的模糊隶属度不满足 “归一性条件” 和 “可加性原则”，导致评估结果的可信度不高 ${ }^{[11]}$ 。 另外，模糊集的 “取大 “、“取小 “运算也会损失 许多有用的信息，造成评估结果不合理。

针对已有研究的不足和风险投资项目高度不确 定性等特点, 结合风险投资运作过程中的实际情 况, 本文从风险投资家的角度出发, 构建风险评价 指标体系, 通过建立两个模型分别解决单个风险投 资项目的风险等级确定问题和多个风险投资项目的 风险排序问题。

\section{2. 评价指标体系的构建}

在借鉴国内外风险投资项目风险评价指标的基 础上, 根据全面性、系统性、可比性、科学性与可 行性相结合的原则 ${ }^{[2,12]}$, 本文考虑将以下几个风险因 素作为风险投资项目风险评价指标:

（1）技术风险 $u_{1}$ : 按新技术生命周期理论 ${ }^{[13]}$, 风险项目由于新思想和新技术本身的先天不足, 技 术不成熟、不完善以及可替代的新技术出现等多种 因素面临的风险。

（2）企业家风险 $u_{2}$ ：指由于委托代理关系引发 的风险企业家逆向选择风险、道德风险、风险投资 家与风险企业家目标差异风险等。

（3）管理风险 $u_{3}$ ：指因管理不善而导致项目 失败的风险。主要是指由于组织制度和结构的科学 性、经营管理方式、管理层能力和经验、约束激励 机制以及人才管理水平等引发的风险。

（4）生产风险 $u_{4}$ : 指与知识产权、生产人员结 构、生产设备水平以及能源原材料供应等相关的风 险。

（5）市场风险 $u_{5}$ ：指新产品、新技术市场竞 争力的不确定性引发的风险。比如市场需求程度、 产品可替代性、产品销售网络、产品生命周期等相 关风险。

（6）投资分析风险 $u_{6}$ ：指与投资工具的选 择、投资规模大小、投资决策方法的科学性、企业
资本结构等方面相关的风险。

\section{3. 单个项目的风险等级评价模型}

当风险投资机构面临一个风险投资项目时，可 以组织多个风险投资家分析风险企业家提交的商业 计划书，根据该项目的具体情况（如所处的行业） 对其风险进行评价, 首先确定该项目的风险评价指 标权重。针对风险投资项目具有较大不确定性的特 点, 本文采用区间数对评价指标间的相对重要性程 度进行描述, 然后利用互补判断矩阵排序向量确定 指标权重。

\section{1 评价指标权重的确定}

记 $\tilde{a}=\left[a^{-}, a^{+}\right]=\left\{x \mid a^{-} \leqslant x \leqslant a^{+}, a^{-}, a^{+} \in R\right\}$, 称 $\tilde{a}$ 为一个区间数 ${ }^{[14]}$ 。

定义 $\mathbf{1}^{[14]}$ 设 $\tilde{a}=\left[a^{-}, a^{+}\right], \tilde{b}=\left[b^{-}, b^{+}\right]$, 且记

$l_{\tilde{a}}=a^{+}-a^{-}, l_{\tilde{b}}=b^{+}-b^{-}$, 则

$p(\tilde{a} \geqslant \tilde{b})=\frac{\min \left\{l_{\tilde{a}}+l_{\tilde{b}}, \max \left(a^{+}-b^{-}, 0\right)\right\}}{l_{\tilde{a}}+l_{\tilde{b}}}$

为 $\tilde{a} \geqslant \tilde{b}$ 的可能度, 且记 $\tilde{a}, \tilde{b}$ 的次序关系为 $\tilde{a} \geqslant \tilde{b}$ 。

正如前面所述，由于风险投资家难以把握影响 风险投资风险的各因素的准确状态，从而会产生主 观上的不确定性。因此, 用传统的层次分析法构造 判断矩阵对评估指标间的相对重要程度进行分析显 然不合理。而不确定层次分析法 (UTAHP) 不需要 风险投资家对各指标的相对重要程度做出精确的判 断, 只需要给出某一估计区间即可。由此, 本文采 用区间数转换判断矩阵中的元素。

首先，根据各风险评估指标间的相互重要性， 构造评估指标的区间数判断矩阵为 $A=\left(A_{i j}\right)_{6 \times 6}$, 其 中 $A_{i j}=\left[a_{i j}^{-}, a_{i j}^{+}\right]=\left\{t \mid 1 / 9 \leqslant a_{i j}^{-} \leqslant t \leqslant a_{i j}^{+} \leqslant 9\right\}$ 为区间数, 表示指标 $u_{i}$ 对 $u_{j}$ 的相对重要程度, 且满足 $a_{i j}^{-}=1 / a_{j i}^{+}, a_{i j}^{+}=1 / a_{j i}^{-}(i, j=1,2, \cdots, 6)$ 。具体方法如 下:

假设有 $n$ 位风险投资家参加评估，任取两个指 标 $u_{i}, u_{j}$, 让每个风险投资家独立给出 $u_{i}$ 与 $u_{j}$ 相对 重要程度的区间数。设第 $h$ 位风险投资家给出的区 间数为: $A_{i j}^{(h)}=\left[a_{i j}^{-(h)}, a_{i j}^{+(h)}\right], \quad(h=1,2, \cdots, n)$ 。则取 $a_{i j}^{-}=\sum_{h=1}^{n} \alpha_{h} a_{i j}^{-(h)}, a_{i j}^{+}=\sum_{h=1}^{n} \alpha_{h} a_{i j}^{+(h)} \quad(1 \leqslant i \leqslant j \leqslant 6)$ 
其中 $\alpha_{h}\left(\sum_{h=1}^{n} \alpha_{h}=1,0<\alpha_{h}<1\right)$ 为第 $h$ 位风险投资家 的重要性程度, 由此得到区间数判断矩阵 $A=\left(A_{i j}\right)_{6 \times 6}$ 。

其次, 设 $A^{-}=\left(a_{i j}^{-}\right)_{6 \times 6}, A^{+}=\left(a_{i j}^{+}\right)_{6 \times 6}$, 则 $A=\left[A^{-}, A^{+}\right]$ 利用某一排序方法（如方根法）对矩阵 $A^{-}, A^{+}$求得 权重向量分别为: $w^{-}=\left(w_{1}^{-}, w_{2}^{-}, \cdots, w_{6}^{-}\right)$, $w^{+}=\left(w_{1}^{+}, w_{2}^{+}, \cdots, w_{6}^{+}\right)$, 取

$$
k=\sqrt{\sum_{j=1}^{6} \frac{1}{\sum_{i=1}^{6} a_{i j}^{+}}}, m=\sqrt{\sum_{j=1}^{6} \frac{1}{\sum_{i=1}^{6} a_{i j}^{-}}}
$$

得到区间数权重向量:

$$
W=\left(W_{1}, W_{2}, \cdots, W_{6}\right)=\left[k w^{-}, m w^{+}\right]
$$

其中

$$
W_{i}=\left[k w_{i}^{-}, m w_{i}^{+}\right] \quad(i=1,2, \cdots, 6)
$$

若 $0<k \leqslant 1 \leqslant m$, 则表明区间数判断矩阵 $A$ 满足一 致性条件, 否则需要反馈给风险投资家重新判断。

最后，利用定义 1 , 对所有区间数权重 $W_{i}=\left[k w_{i}^{-}, m w_{i}^{+}\right] \quad(i=1,2, \cdots, 6)$ 两两进行比较, 建立可 能度矩阵 $P=\left(p_{i j}\right)_{6 \times 6}$, 其中 $p_{i j}=p\left(W_{i} \geqslant W_{j}\right)$ 。由可能 度的性质可知, 可能度矩阵 $P$ 是一个互补判断矩 阵, 利用互补判断矩阵排序向量计算公式 [14], 可求 出各评估指标的权重为:

$$
\begin{aligned}
& w_{i}=\frac{1}{q(q-1)}\left(\sum_{j=1}^{q} p_{i j}+\frac{q}{2}-1\right) \quad(i=1,2, \cdots, q) \\
& \text { (在本文中 } q=6 \text { ) }
\end{aligned}
$$

\section{2 基于EWAA算子的风险等级评价}

由于风险投资是一种创新投资, 项目缺乏历史 数据, 因此, 专家往往利用语言形式对项目的风险 评估指标进行评估。现将各评估指标按照其风险状 况划分为 9 个等级, 相应的语言标度集为

$S=\left\{S_{-4}=\right.$ 极低、 $S_{-3}=$ 很低、 $S_{-2}=$ 低、 $S_{-1}=$ 较 低、 $S_{0}=$ 一般、 $S_{1}=$ 较高、 $S_{2}=$ 高、 $S_{3}=$ 很高、 $S_{4}=$ 极高 $\}$ 。

为了便于计算和避免丢失决策信息, 在原有语言 标度 $S=\left\{s_{\beta} \mid \beta=-4, \cdots 4\right\}$ 的基础上定义一个拓展 语言标度 $\bar{S}=\left\{s_{\bar{\beta}} \mid \bar{\beta} \in[-l, l]\right\}$, 其中 $l$ 是一个充分
大的自然数, $\bar{\beta}=\sum_{i=1}^{q} w_{i} \beta_{i}, w=\left(w_{1}, w_{2}, \cdots w_{q}\right)$ 是 语言数据 $s_{\beta_{i}}(i=1,2, \cdots, q)$ 的加权向量。

定义 $2^{[14]}$ 假设 EWAA: $S \rightarrow \bar{S}$, 令

$$
\operatorname{EWAA}_{w}\left(s_{\beta_{1}}, s_{\beta_{2}}, \cdots, s_{\beta_{q}}\right)=w_{1} s_{\beta_{1}} \oplus w_{2} s_{\beta_{2}} \oplus \cdots
$$

$$
\oplus w_{n} s_{\beta_{q}}=s_{\bar{\beta}}
$$

称函数 EWAA 为扩展的加权算术平均算子。

假设第 $\mathrm{h}$ 位风险投资家对该项目的第 $i$ 个风险评 价指标的语言评估值为

$$
s_{\beta_{i}}^{h}(i=1,2, \cdots, 6, h=1,2, \cdots, n),
$$

利用(6)式对以上指标评估信息进行集结可得第 $\mathrm{h}$ 位 风险投资家对该项目的风险语言评估值为:

$$
s_{\bar{\beta}^{h}}=w_{1} s_{\beta_{1}}^{h} \oplus w_{2} s_{\beta_{2}}^{h} \oplus \cdots \oplus w_{6} s_{\beta_{6}}^{h}
$$

其中 $\bar{\beta}^{h}=\sum_{i=1}^{6} w_{i} \beta_{i}$ 。

进一步利用(6)式对这 $n$ 位风险投资家的项目风 险评估信息进行集结，得到该风险投资项目的风险 语言评估值为

$$
s_{b}=\alpha_{1} S_{\bar{\beta}^{1}} \oplus \alpha_{2} S_{\bar{\beta}^{2}} \oplus \cdots \oplus \alpha_{n} s_{\bar{\beta}^{n}}
$$

其中

$$
\begin{aligned}
b= & \sum_{h=1}^{n} \alpha_{h} \bar{\beta}^{h}(-4 \leqslant u \leqslant b \leqslant v \leqslant 4, v-u=1, \\
& u, v \in Z) 。
\end{aligned}
$$

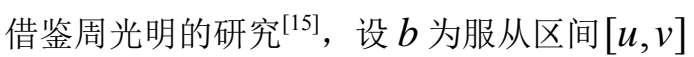
上均匀分布的随机变量的取值, 则可得到该项目风 险等级的评估准则:

(1) 若 $0 \leqslant b-u \leqslant 0.5$, 则该项目的风险在置信度 $(1+u-b) \%$ 下的风险等级为 $S_{u}$;

(2) 若 $0 \leqslant v-b \leqslant 0.5$, 则该项目的风险在置信度 $(1+b-v) \%$ 下的风险等级为 $S_{v}$ 。

从以上评估准则可以看出, 本文构建的模型与 已有研究的不同之处在于: 在一定置信度下确定风 险投资项目的风险等级, 避免了评估结果的绝对 化，从而更加符合风险投资项目风险评估的实际。

\section{4. 多个项目的风险排序模型}

从第 3 节的风险评价模型可知, 针对单个风险 投资项目的风险评价方法最终能够在一定置信度下 
确定其风险等级。然而风险投资家在风险投资的实 际运作中, 经常需要对多个风险投资项目的风险大 小进行比较, 从而根据风险程度进行项目选择。一 个很自然的想法是, 按照第 3 节的方法, 对每个项 目的风险进行评价, 然后比较其风险大小。但是, 这样的方法可能面临一个现实的问题: 单项目的风 险评价方法没有得到项目风险的具体评分值, 只是 确定了其风险等级, 因此, 如果有若干个项目的风 险等级评价结果相同, 那么该如何选择项目呢? 为 了解决这个现实问题, 有必要对多个风险投资项目 的风险评价方法进行研究。

\section{1 不确定多属性决策模型}

由于只是解决多个风险投资项目的风险排序问 题和为了计算的方便, 现将风险投资项目评价指标 的风险划分为五个等级：低风险 $V_{1}$, 较低风险 $V_{2}$, 一般风险 $V_{3}$, 较高风险 $V_{4}$, 高风险 $V_{5}$ 。以上五个 评价等级元素构成等级集合: $V=\left\{V_{1}, V_{2}, V_{3}, V_{4}, V_{5}\right\}$, 按照 5 分制打分, 五个风险等级对应的值分别赋予 为 $1 、 2 、 3 、 4 、 5$ 。若指标等级介于两相邻等级之 间，则相应评分值为 $1.5 、 2.5 、 3.5 、 4.5$ 。

下面建立一个基于区间型理想点的不确定多属 性决策模型用于解决多个风险投资项目的风险排序 问题。

定义 $3^{[14]}$ 设区间数 $\tilde{a}=\left[a^{-}, a^{+}\right], \tilde{b}=\left[b^{-}, b^{+}\right]$, 令 范数 $\|\tilde{a}-\tilde{b}\|=\sqrt{\left(b^{-}-a^{-}\right)^{2}+\left(b^{+}-a^{+}\right)^{2}}$, 称 $d(\tilde{a}, \tilde{b})=\|\tilde{a}-\tilde{b}\|$ 为区间数 $\tilde{a}, \tilde{b}$ 的相离度。

显然 $d(\tilde{a}, \tilde{b})$ 越大, 则区间数 $\tilde{a}, \tilde{b}$ 相离的程度 就越大。特别地, 当 $d(\tilde{a}, \tilde{b})=0$ 时, 有 $\tilde{a}=\tilde{b}$, 即 区间数 $\tilde{a}, \tilde{b}$ 相等。利用定义 3 可以衡量两个区间数 相似的程度。

现假设有 $s$ 个风险投资项目 $x_{1}, x_{2}, \cdots, x_{s}$ 可供风 险投资家选择，评价指标为 $u_{1}, u_{2}, \cdots, u_{6}$, 风险投资 家对这些项目的风险评价指标按照5分制打分。如前 所述，风险投资家所掌握的信息不足以把握评价指 标的真实状态, 以区间数的形式给出指标判断值, 从而得到项目决策矩阵为 $\tilde{A}=\left(\tilde{a}_{i j}\right)_{s \times 6}$, 其中 $\tilde{a}_{i j}=\left[a_{i j}^{-}, a_{i j}^{+}\right]=\left\{t \mid 1 \leqslant a_{i j}^{-} \leqslant t \leqslant a_{i j}^{+} \leqslant 5\right\}$ 表示第 $i$ 个项目的第 $j$ 个指标风险等级区间数 $(i=1,2, \cdots, s, j=1,2, \cdots, 6)$ 。
风险投资项目风险评价指标都为成本型指标， 为了消除不同物理量纲对决策结果的影响, 可用以 下公式 ${ }^{[14]}$ 将决策矩阵 $\tilde{A}=\left(\tilde{a}_{i j}\right)_{s \times 6}$ 转化为规范化决策矩 阵 $\tilde{R}=\left(\tilde{r}_{i j}\right)_{s \times 6}$, 其中

$$
\begin{gathered}
\tilde{r}_{i j}=\left[r_{i j}^{-}, r_{i j}^{+}\right]=\left\{t \mid 0 \leqslant r_{i j}^{-} \leqslant t \leqslant r_{i j}^{+} \leqslant 1\right\} \\
(i=1,2, \cdots, s, j=1,2, \cdots, 6) 。 \\
r_{i j}^{-}=\frac{1 / a_{i j}^{+}}{\sqrt{\sum_{i=1}^{n}\left(1 / a_{i j}^{-}\right)^{2}}}, \quad r_{i j}^{+}=\frac{1 / a_{i j}^{-}}{\sqrt{\sum_{i=1}^{n}\left(1 / a_{i j}^{+}\right)^{2}}} \\
(i=1,2, \cdots, s, j=1,2, \cdots, 6)
\end{gathered}
$$

定义 4 称 $\tilde{r}=\left(\tilde{r}_{1}^{*}, \tilde{r}_{2}^{*}, \cdots, \tilde{r}_{6}^{*}\right)$ 为区间型理想点, 其 中

$$
\tilde{r}_{j}^{*}=\left[\tilde{r}_{j}^{-*}, \tilde{r}_{j}^{+^{*}}\right]=\left[\max _{i}\left(r_{i j}^{-}\right), \max _{i}\left(r_{i j}^{+}\right)\right](j=1,2, \cdots, 6)
$$

不确定多属性决策方法的实质是利用已有的决 策信息通过一定的方式对一组（有限个）备选方案 进行排序并择优。在处理风险投资项目风险评价问 题时，用区间数表示指标值较为合理，因此，可以 建立指标权重未知且指标值为区间数的不确定多属 性决策模型对多个风险投资项目的风险进行排序。

由规范化矩阵 $\tilde{R}=\left(\tilde{r}_{i j}\right)_{s \times 6}$ 、指标权重向量 $w=\left(w_{1}, w_{2}, \cdots, w_{6}\right)^{T}$ 及区间数的运算法则可知，风险 投资项目 $x_{i}$ 的风险综合属性值为

$$
\tilde{z}_{i}=\sum_{j=1}^{6} \tilde{r}_{i j} w_{j}=\left[\sum_{j=1}^{6} r_{i j}^{-} w_{j}, \sum_{j=1}^{6} r_{i j}^{+} w_{j}\right] \quad(i=1,2, \cdots, s)
$$

式中 $w_{j}$ 为第 $j$ 个指标 $u_{j}$ 的未知权重, 满足单位 化约束条件 $\sum_{j=1}^{6} w_{j}^{2}=1\left(w_{j} \geqslant 0 j=1,2, \cdots, 6\right)$ 。

因为风险评价指标权重向量 $w=\left(w_{1}, w_{2}, \cdots, w_{6}\right)^{T}$ 完全未知, 所以不能直接由(11)式确定项目风险综合 属性值。

徐泽水 ${ }^{[16]}$ 针对只有部分权重信息且指标值为实 数的不确定多属性决策问题, 提出过一种基于方案 满意度的决策方法。该研究指出对于每个方案 $x_{i}(i=1,2, \cdots, s)$ 而言, 其满意度总是越大越好。利 用该研究思想，本文构建基于区间型理想点的不确 定多属性决策模型解决多个风险投资项目风险评价 问题。

对于风险投资项目 $x_{i}$ 而言, 它越接近区间型理 想点就越优。因此, 可令项目 $x_{i}$ 与区间型理想点 
$\tilde{r}=\left(\tilde{r}_{1}^{*}, \tilde{r}_{2}^{*}, \cdots, \tilde{r}_{6}^{*}\right)$ 之间的加权偏差为

$$
\begin{gathered}
D_{i}(w)=\sum_{j=1}^{6}\left\|\tilde{r}_{i j}-\tilde{r}_{j}^{*}\right\| w_{j}=\sum_{j=1}^{6} d\left(\tilde{r}_{i j}, \tilde{r}_{j}^{*}\right) w_{j} \\
(i=1,2, \cdots, s)
\end{gathered}
$$

对于给定的指标权重向量 $w=\left(w_{1}, w_{2}, \cdots, w_{6}\right)^{T}$, $D_{i}(w)$ 越小则项目 $x_{i}$ 越优。因此可建立如下的多目 标决策模型:

$$
\left\{\begin{array}{l}
\min D(w)=\left(D_{1}(w), D_{2}(w), \cdots, D_{s}(w)\right) \\
s . t \sum_{j=1}^{6} w_{j}^{2}=1 \quad w_{j} \geqslant 0 j=1,2, \cdots, 6
\end{array}\right.
$$

由于每个项目之间是公平竞争，不存在任何偏 好关系，因此，上述多目标决策模型可用等权线性 加权法综合为如下等价的单目标最优化模型:

$$
\left\{\begin{array}{l}
\min D(w)=\sum_{i=1}^{s} D_{i}(w)=\sum_{i=1}^{s} \sum_{j=1}^{6} d\left(\tilde{r}_{i j}, \tilde{r}_{j}^{*}\right) w_{j} \\
\text { s.t } \sum_{j=1}^{6} w_{j}^{2}=1 \quad w_{j} \geqslant 0 j=1,2, \cdots, 6
\end{array}\right.
$$

求解该模型得到

$$
w_{j}=\frac{\sum_{i=1}^{s} d\left(\tilde{r}_{i j}, \tilde{r}_{j}^{*}\right)}{\sqrt{\sum_{j=1}^{6}\left(\sum_{i=1}^{s} d\left(\tilde{r}_{i j}, \tilde{r}_{j}^{*}\right)\right)^{2}}}(j=1,2, \cdots, 6)
$$

将上述权重向量 $w=\left(w_{1}, w_{2}, \cdots, w_{6}\right)^{T}$ 作归一化处理, 可得

$$
w_{j}^{*}=\frac{\sum_{i=1}^{s} d\left(\tilde{r}_{i j}, \tilde{r}_{j}^{*}\right)}{\sum_{j=1}^{6} \sum_{i=1}^{s} d\left(\tilde{r}_{i j}, \tilde{r}_{j}^{*}\right)} \quad(j=1,2, \cdots, 6)
$$

求出最优权重向量 $w^{*}=\left(w_{1}^{*}, w_{2}^{*}, \cdots, w_{6}^{*}\right)^{T}$ 之后, 通 过(11)式计算出各个项目的风险综合属性值 $\tilde{z}_{i}(i=1,2, \cdots, s)$ 。由于 $\tilde{z}_{i}(i=1,2, \cdots, s)$ 仍然是区间 数, 不便于直接对项目风险进行排序, 因此可利用 (1)式计算出 $\tilde{z}_{i}(i=1,2, \cdots, s)$ 之间的可能度, 并建立 可能度矩阵 $P=\left(p_{i k}\right)_{s \times s}$, 其中 $p_{i k}=p\left(\tilde{z}_{i} \geqslant \tilde{z}_{k}\right)$ 。 $(i, k=1,2, \cdots, s)$ 矩阵 $P$ 是一个互补判断矩阵, 利 用(5)式得到矩阵 $P$ 的排序向量

$$
\omega=\left(\omega_{1}, \omega_{2}, \cdots, \omega_{s}\right)^{T}
$$

并按其分量大小对区间数 $\tilde{z}_{i}(i=1,2, \cdots, s)$ 进行排序, 由此可得到风险投资项目 $x_{1}, x_{2}, \cdots, x_{s}$ 的风险排 序。

\section{5. 算例分析}

\section{1 单项目风险等级评价算例}

假设三个风险投资家对某一风险投资项目的风 险进行评估, 评估指标如第 2 节所述。

不妨设三个风险投资家的重要性程度相同，按 照3.1节所述方法，对该三个风险投资家的区间数判 断矩阵进行处理（具体计算过程略），得到区间数 判断矩阵 $A=\left(A_{i j}\right)_{6 \times 6}$ 。

$$
A=\left(\begin{array}{ccc}
1 & {[2.33,3.33]} & {[2.00,3.00]} \\
{[0.30,0.43]} & 1 & {[0.33,0.50]} \\
{[0.33,0.50]} & {[2.00,3.03]} & 1 \\
{[0.27,0.37]} & {[0.33,0.50]} & {[0.33,0.50]} \\
{[2.00,3.03]} & {[3.03,4.00]} & {[2.00,3.03]} \\
{[0.23,0.30]} & {[0.30,0.43]} & {[0.27,0.37]}
\end{array}\right.
$$

$$
\begin{array}{ccc}
{[2.67,3.67]} & {[0.33,0.50]} & {[3.33,4.33]} \\
{[2.00,3.00]} & {[0.25,0.33]} & {[2.33,3.33]} \\
{[2.00,3.00]} & {[0.33,0.50]} & {[2.67,3.67]} \\
1 & {[0.20,0.33]} & {[2.00,3.00]} \\
{[3.03,5.00]} & 1 & {[3.67,5.33]} \\
{[0.33,0.50]} & {[0.19,0.27]} & 1
\end{array}
$$

贝刂

$$
A^{-}=\left(\begin{array}{cccccc}
1 & 2.33 & 2.00 & 2.67 & 0.33 & 3.33 \\
0.30 & 1 & 0.33 & 2.00 & 0.25 & 2.33 \\
0.33 & 2.00 & 1 & 2.00 & 0.33 & 2.67 \\
0.27 & 0.33 & 0.33 & 1 & 0.20 & 2.00 \\
2.00 & 3.03 & 2.00 & 3.03 & 1 & 3.67 \\
0.23 & 0.30 & 0.27 & 0.33 & 0.19 & 1
\end{array}\right)
$$




$$
A^{+}=\left(\begin{array}{cccccc}
1 & 3.33 & 3.00 & 3.67 & 0.50 & 4.33 \\
0.43 & 1 & 0.50 & 3.00 & 0.33 & 3.33 \\
0.50 & 3.03 & 1 & 3.00 & 0.50 & 3.67 \\
0.37 & 0.50 & 0.50 & 1 & 0.33 & 3.00 \\
3.03 & 4.00 & 3.03 & 5.00 & 1 & 5.33 \\
0.30 & 0.43 & 0.37 & 0.50 & 0.27 & 1
\end{array}\right)
$$

利用方根法求出 $A^{-}, A^{+}$的归一化权重向量分别 为:

$$
\begin{aligned}
& w^{-}=(0.231,0.127,0.169,0.095,0.307,0.071) \\
& w^{+}=(0.227,0.126,0.171,0.097,0.310,0.069)
\end{aligned}
$$

由(2)式得到: $k=0.913<1, m=1.103>1$, 因此 区间数判断矩阵 $A$ 满足一致性要求。

$$
\begin{aligned}
& \text { 由(3)式得到: } \\
& W_{1}=[0.211,0.250], W_{2}=[0.116,0.139], \\
& W_{3}=[0.154,0.189], W_{4}=[0.087,0.107], \\
& W_{5}=[0.280,0.342], W_{6}=[0.065,0.076]
\end{aligned}
$$

由(1)式计算出各区间数权重之间的可能度, 并 建立可能度矩阵为:

$$
P=\left(\begin{array}{cccccc}
0.5 & 1 & 1 & 1 & 0 & 1 \\
0 & 0.5 & 0 & 1 & 0 & 1 \\
0 & 1 & 0.5 & 1 & 0 & 1 \\
0 & 0 & 0 & 0.5 & 0 & 1 \\
1 & 1 & 1 & 1 & 0.5 & 1 \\
0 & 0 & 0 & 0 & 0 & 0.5
\end{array}\right)
$$

由(5)式求出各个评估指标的权重为: $w=(0.217,0.150,0.183,0.117,0.250,0.083)$

现假设这三个风险投资家对该项目评估指标的 评估值如表 1 所示:

表 1 三位专家对该风险项目非系统风险的评估值

\begin{tabular}{l|llllll}
\hline & $u_{1}$ & $u_{2}$ & $u_{3}$ & $u_{4}$ & $u_{5}$ & $u_{6}$ \\
\hline$d_{1}$ & $s_{-2}$ & $s_{1}$ & $s_{-2}$ & $s_{0}$ & $s_{-3}$ & $s_{1}$ \\
$d_{2}$ & $s_{-1}$ & $s_{-1}$ & $s_{-1}$ & $s_{0}$ & $s_{0}$ & $s_{0}$ \\
$d_{3}$ & $s_{-2}$ & $s_{1}$ & $s_{-1}$ & $s_{-1}$ & $s_{-2}$ & $s_{1}$ \\
\hline
\end{tabular}

表1中 $d_{j}(j=1,2,3)$ 表示第 $j$ 位专家。
利用(7)式对以上指标评估信息进行集结，得到 这三个风险投资家对该项目风险的语言评估值分别 为:

$$
S_{\bar{\beta}^{1}}=S_{-1.317}, S_{\bar{\beta}^{2}}=S_{-0.550}, S_{\bar{\beta}^{3}}=S_{-1.001}
$$

再利用(8)式对这三个风险投资家的评估信息进 行集结, 得到该项目非系统风险的语言评估值为

$$
S_{b}=S_{-0.8703^{\circ}} \text {. }
$$

根据单项目风险等级评估准则(1)可知, 该项目 的风险在置信度 $87.03 \%$ 下的风险等级为 $S_{-1}$, 即该 项目的风险在置信度 $87.03 \%$ 下处于 “较低” 等 级。

\section{2 多项目风险排序算例}

假设现有 5 个备选项目 $x_{1}, x_{2}, \cdots, x_{5}$ 可供风险投 资家进行选择，风险投资家从项目风险因素角度对 这些项目进行评价, 评价指标如第 2 节所述。评价 对象空间为: $X=\left\{x_{1}, x_{2}, \cdots, x_{5}\right\}$ ， 评价指标空间为: $U=\left\{u_{1}, u_{2}, \cdots, u_{6}\right\}$ 。得到项目 决策矩阵 $\tilde{A}=\left(\tilde{a}_{i j}\right)_{5 \times 6}$ 为:

$$
\tilde{A}=\left(\begin{array}{llllll}
{[3,4]} & {[3,4]} & {[2,3]} & {[2,3]} & {[2,4]} & {[4,5]} \\
{[2,3]} & {[3,4]} & {[4,5]} & {[2,4]} & {[3,4]} & {[2,3]} \\
{[2,3]} & {[3,4]} & {[4,5]} & {[2,4]} & {[2,3]} & {[3,5]} \\
{[2,4]} & {[2,5]} & {[2,3]} & {[3,4]} & {[3,5]} & {[2,3]} \\
{[3,4]} & {[2,5]} & {[2,4]} & {[3,5]} & {[4,5]} & {[2,4]}
\end{array}\right)
$$

将决策矩阵 $\tilde{A}$ 按(9)式规范化以后得到规范化决 策矩阵 $\tilde{R}=\left(\tilde{r}_{i j}\right)_{5 \times 6}$ 如下:

$$
\begin{aligned}
\tilde{R}= & \left(\begin{array}{llll}
{[0.254,0.521]} & {[0.274,0.645]} & {[0.357,0.828]} \\
{[0.338,0.781]} & {[0.274,0.645]} & {[0.214,0.414]} \\
{[0.338,0.781]} & {[0.274,0.645]} & {[0.214,0.414]} \\
{[0.254,0.781]} & {[0.219,0.967]} & {[0.357,0.828]} \\
{[0.254,0.521]} & {[0.219,0.967]} & {[0.267,0.828]} \\
& {[0.338,0.859]} & {[0.282,0.889]} & {[0.208,0.414]} \\
& {[0.254,0.859]} & {[0.282,0.593]} & {[0.347,0.828]} \\
& {[0.254,0.859]} & {[0.376,0.889]} & {[0.208,0.552]} \\
& {[0.254,0.573]} & {[0.226,0.593]} & {[0.347,0.828]} \\
& {[0.203,0.573]} & {[0.226,0.445]} & {[0.260,0.828]}
\end{array}\right) \\
& \text { 利用(16)式可以求得最优权重向量为 }
\end{aligned}
$$

$w^{*}=(0.115,0.196,0.176,0.142,0.219,0.152)^{T}$ 利用(11)式求得各项目的风险综合属性值: 
$\tilde{z}_{1}(w)=[0.287,0.712], \tilde{z}_{2}(w)=[0.281,0.667]$,

$\tilde{z}_{3}(w)=[0.303,0.730], \tilde{z}_{4}(w)=[0.263,0.737]$,

$\tilde{z}_{5}(w)=[0.237,0.635]$ 。

由(1)式计算出各项目综合属性值之间的可能

度, 并建立可能度矩阵为:

$$
P=\left(\begin{array}{ccccc}
0.5 & 0.545 & 0.490 & 0.509 & 0.586 \\
0.455 & 0.5 & 0.444 & 0.466 & 0.546 \\
0.510 & 0.556 & 0.5 & 0.518 & 0.598 \\
0.491 & 0.534 & 0.482 & 0.5 & 0.573 \\
0.414 & 0.454 & 0.402 & 0.427 & 0.5
\end{array}\right)
$$

由(5)式计算得到可能度矩阵 $P$ 的排序向量为: $\omega=(0.207,0.196,0.209,0.204,0.185)^{T}$

由排序向量 $\omega$ 及矩阵 $P$ 中的可能度, 得到区间 数 $\tilde{z}_{i}(w)(i=1,2, \cdots, 5)$ 的排序:

$$
\tilde{z}_{3}(w) \underset{0.510}{\succ} \tilde{z}_{1}(w) \underset{0.509}{\succ} \tilde{z}_{4}(w) \underset{0.534}{\succ} \tilde{z}_{2}(w) \succ_{0.546} \tilde{z}_{5}(w)
$$

因此这 5 个风险投资项目的排序为 (从风险角度 考虑）：

$$
x_{3} \succ_{0.510}^{\succ} x_{1} \succ_{0.509} x_{4} \succ_{0.534}^{\succ} x_{2} \succ_{0.546}^{\succ} x_{5} .
$$

故最优项目为 $X_{3}$ 。

\section{6. 结束语}

在借鉴国内外风险投资项目风险评价指标的基 础上, 本文建立了符合我国实际情况的风险投资项 目风险评价指标体系, 针对单个风险投资项目的风 险等级确定问题和多个风险投资项目的风险排序问 题, 分别构建模型予以解决。在单项目风险评价模 型的构建中，充分考虑了风险投资项目和专家主观 认识上的不确定性, 采用区间数对风险投资项目的 风险评估指标相对重要性程度进行描述。本文构建 的模型能够在一定置信度下确定风险投资项目的风 险等级, 避免了传统评估方法所得结果的绝对化。 在多项目风险评价模型的构建中, 同样用区间数来 描述风险投资项目风险评价指标的风险等级, 构建 了基于区间型理想点的不确定多属性决策模型，从 而实现对多个风险投资项目进行风险排序。由于充 分认识到风险投资项目和专家主观认识上的不确定 性, 而采用区间数来进行刻画, 因此本文构建的风 险评价模型符合风险投资的特点, 具有较好的科学 性和合理性。

\section{参考文献}

[1] 成思危. 成思危论风险投资. 北京: 中国人民大学出版 社, 2008 .

[2] Tyebjee T T, B runo A V. A model of ven ture capitalist investment activity . Management Science, 1 984,30(9): 1051-1066.

[3] Wright M. R obbie K. Venture capital. Bookfiled : Dartmouth Publishing Company Ltd. 1997.

[4] Koski T M.Using fuzzy set theory in assessing the success potential of venture capital investment theory and pilot field study.International Journal of Entrepreneurship and Innovation Management,2003,3(5):509-524.

[5] Cheng $\mathrm{CH}, \mathrm{Y}$ ang K L, Hwang C L. Evaluating attack helicopters b y AHP based on linguistic vari able weight. European Journal of Operational Research, 199 9, 116(2):423-435.

[6] 钱水土,周春喜. 风险投资的风险综合评价研究. 数量经 济技术经济研究,2002,(5):45-48.

[7]徐宪平. 风险投资的风险评价与控制.中国管理科 学, 2001,9 (4):75- 80.

[8] 万玉成,盛昭瀚. 基于未确知测度的风险投资非系统风险 的评价与控制研究. 系统工程理论与实践,24(11):22-27.

[9] 赵振武,唐万生,宁玉富. 基于模糊模拟的 AHP 在风险投 资项目评价中的应用. 模糊系统与数学, 20(4):128-133.

[10] 刘德学,㚞治平,王欣荣. 风险投资项目非系统风险的分析 与评价. 系统工程理论方法应用,11(3)198-201.

[11] 刘开第,庞彦军,吴和琴等. 模糊隶属度定义中隐含的问题. 系统工程理论与实践,2000,20(1):110- 112 .

[12] 萧维嘉,全允桓,王剑雨. 基于阶段门的初创企业评价指标 差异性研究. 中国软科学,2008,(12):121-128

[13] 周宗放等. 新兴技术企业信用风险评价理论与方法. 北京: 科学出版社, 2010.

[14] 徐泽水.不确定性多属性决策方法及应用. 北京:清华大学 出版社, 2004 .

[15] 周光明,刘树人.不确定多属性决策中区间数的一种新排 序法.系统工程,2006,24(4):115-117.

[16] 徐泽水,孙在东. 一种基于方案满意度的不确定多属性决 策方法. 系统工程,2001,19(3):76-79. 